\title{
Congenital melanocytic nevus and Fibroepithelioma of Pinkus: a rare type of collision tumor
}

\author{
Bhatta AK ${ }^{1,2}$, Keyal U1,2, Liu $Y^{1}$ \\ ${ }^{1}$ Department of Dermatopathology, Shanghai Skin Disease Hospital, Tongji University School of Medicine, Shanghai, \\ China; ${ }^{2}$ Department of Dermatology, Nepal Mediciti Hospital, Kathmandu, Nepal
}

\begin{abstract}
Congenital melanocytic nevus is a benign pigmented lesion of the skin usually present at birth. Here we report a case of a 61-year-old male who presented with a pigmented lesion on the trunk, which on biopsy showed features of both congenital melanocytic nevus and fibroepithelioma of Pinkus. We reviewed the literature for this combination and found only one case reported so far. Hence, it seems that it is very unusual to see fibroepithelioma of Pinkus in conjunction with a melanocytic lesion.
\end{abstract}

Key words: Collusion tumor; Congenital Melanocytic Nevus; Fibroepithelioma of Pinkus

\section{Dear Editor,}

Congenital melanocytic nevus (CMN) is benign pigmented lesion of the skin usually present at birth. All CMN, regardless of size, may be precursors of malignant melanoma. Fibroepithelioma of Pinkus (FEP) is considered as an unusual variant of basal cell carcinoma (BCC). Here we report a case of 61-year-old male who presented with a pigmented lesion on the trunk, which on biopsy showed features of both CMN and FEP. We reviewed literature for this combination and found only one case reported so far. ${ }^{1}$ Hence, it seems that it is very unusual to see FEP in conjunction with a melanocytic lesion. So, we report this case because of its atypical histopathologic presentation.

A 61-year-old Chinese male presented with a black colored single lesion on left lower abdomen, which was there since birth. The lesion had grown proportionately with the growth of the body. As stated by the patient; the surface of lesion had become more and more rough as it grows bigger and it had become thicker and elevated with time. Recently, patient noticed the change in color of lesion from black to grey, which made him to seek medical advice. There were no

Funding: This study was funded by grants from Shanghai hospital development center project (SHDC12014217) and Science and Technology commission of Shanghai municipality. (16411961500)

Conflict of Interest: No

\section{Address of Correspondence}

Prof. Yeqiang Liu, MD PhD

Department of Dermatopathology, Shanghai Skin Disease Hospital, Shanghai, China.

Telphone: +86-18017336841

E-mail: lyqdoctor@163.com associated symptoms like pain, itch or bleeding. On physical examination, a sharply bordered, unequally colored pigmented lesion, measuring about $7 \mathrm{~cm} \mathrm{x}$ $5 \mathrm{~cm}$ was observed in the left lower abdomen (Figure 1a). The color of lesion was darker in center than periphery. The surface appeared textured and there were few hairs sparsely distributed in the periphery of the lesion. On palpation, it appeared heterogeneous in consistency, which was relatively hard in the core. Lymph nodes were not enlarged. Systemic examination revealed no abnormality. No other family members had similar lesions. A provisional diagnosis of congenital melanocytic nevus was made and in view of its malignant potential, a biopsy $(5 \mathrm{~mm}$ punch biopsy from the core of the lesion) was performed to rule out melanoma.

Histopathology showed nests of melanocytes in dermal stroma and diffuse infiltration of melanocytes in perivascular area, adnexal structures and dermoepidermal junction, which confirmed the diagnosis of congenital melanocytic nevus. In addition, the tumor showed a fenestrated pattern

Submitted: $9^{\text {th }}$ December, 2020

Accepted: $27^{\text {th }}$ January, 2021

Published: $20^{\text {th }}$ February 2021

How to cite this article

Bhatta AK, Keyal U, Liu Y. Congenital melanocytic nevus and Fibroepithelioma of Pinkus: a rare type of collision tumor. Nepal Journal of Dermatology, Venereology \& Leprology 2021;19(1):81-3. https://doi.org/10.3126/njdvl.v19i1.35955.

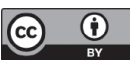

Licensed under CC BY 4.0 International License which permits use, distribution and reproduction in any medium, provided the original work is properly cited. 
of anastomosing epithelial strands and significant dermal fibrosis. The fenestrated portion of the lesion had a blunt interface with underlying dermal stroma suggestive of fibroepithelioma of Pinkus (Figure 1b, 1c). On the basis of these findings, a confirmatory diagnosis of CMN in conjunction with FEP was made.

Patient was counseled about malignant potential of CMN and about possible association of FEP with gastrointestinal(GI)cancer. Surgical excision of thelesion was advised but patient denied any treatment until he suffers any discomfort or experience any symptoms. However, we performed immunohistochemistry (IHC) to further confirm the absence of melanoma. IHC for HMB45, Melan A, S100, MITF and EmA were all negative (Figure 2a-e). Also, patient was convinced to make follow up visits every year. At one year follow up,

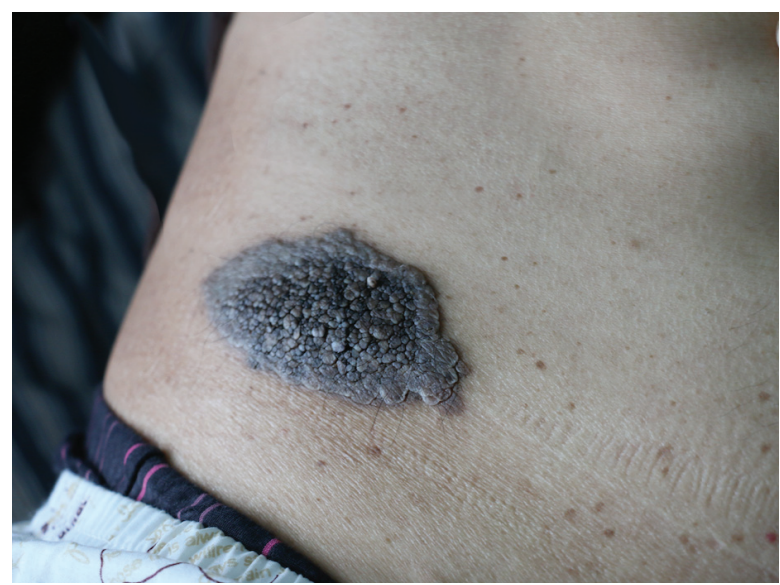

Figure 1a: sharply demarcated, gray-black colored lesion with verrucous hairy surface, about $7 \mathrm{~cm} * 5 \mathrm{~cm}$ in left lower abdomen.

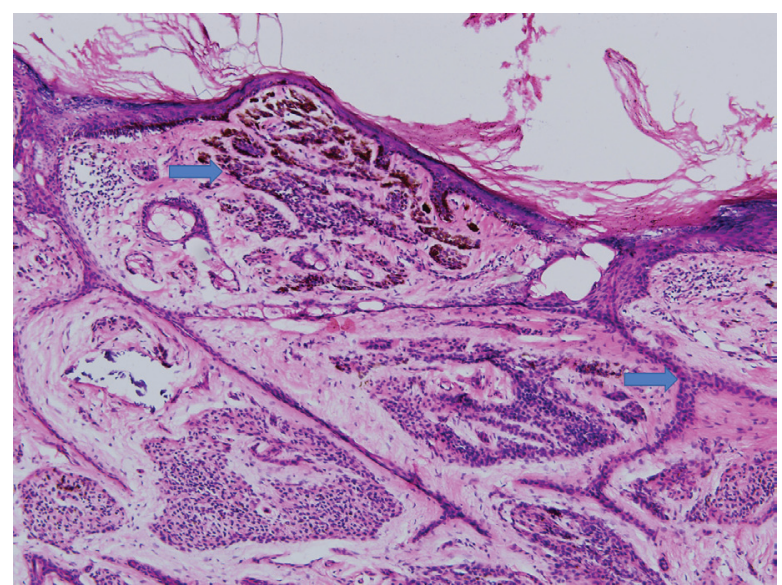

Figure 1c: Fenestrated portion of the tumor shows thin anastomosing strands of basaloid epithelial cells projecting down from the epithelium and dividing fibrous dermal stroma suggestive of FEP. In addition, diffuse infiltration of melanocytes in perivascular and adnexal structures is seen suggestive of CMN (HE 100x) patient was comfortable with no new changes in the lesion, and no any symptoms were reported.

FEP has been reported in the literature in association with many diseases, such as Paget's disease ${ }^{2}$, breast cancer ${ }^{3,4} \mathrm{GI}$ cancer $^{5}$, and, as a variant of BCC, Gorlin-Goltz syndrome. ${ }^{6}$ Also, one of the review paper on FEP has mentioned that it shares characteristics of both trichoblastoma and BCC and has concluded that FEP is an intermediate form between the benign trichoblastoma and the malignant BCC. ${ }^{7}$

It has also been reported as a part of collision tumor with combined melanocytic lesion. ${ }^{1}$ Collision tumors are rare tumors composed of two histologically distinct neoplasms coinciding at the same location. Here we report a case, which on initial presentation simply

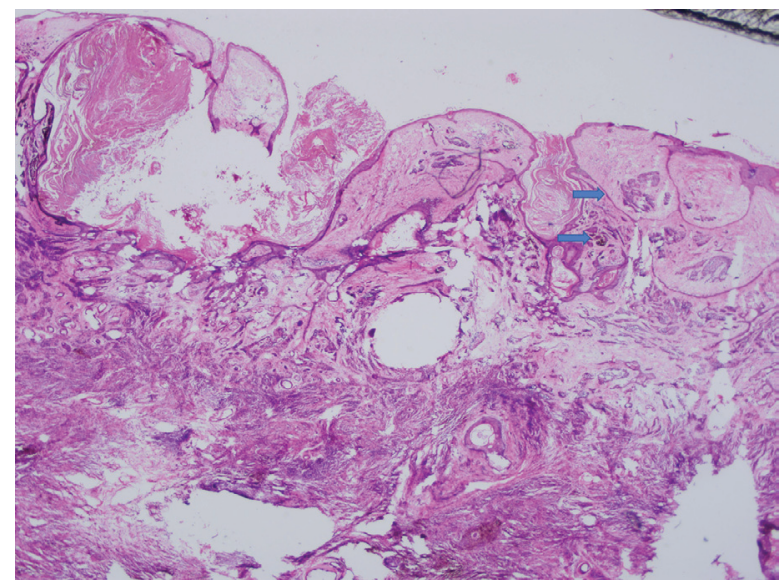

Figure 1b: Nests of melanocytes and some melanocytes in perivascular, adnexal structures, and in dermoepidermal junction with significant dermal fibrosis (HE 40X)

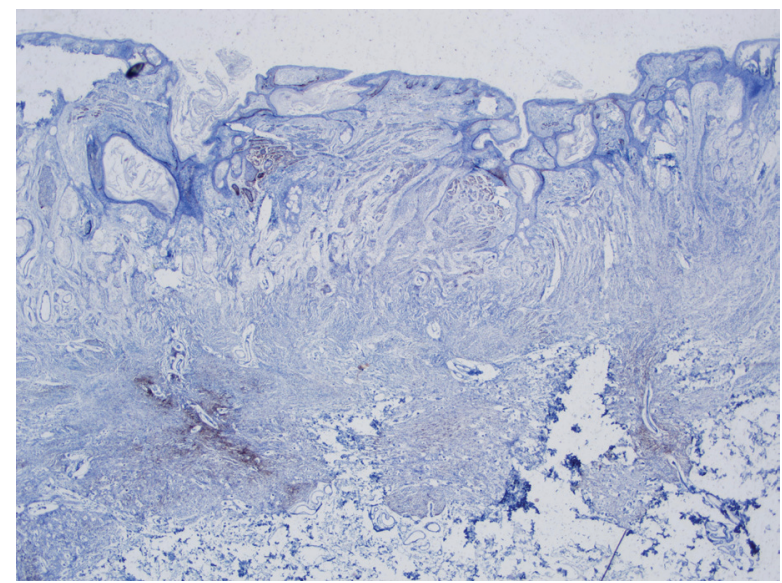

Figure 2a: IHC showing negative stain for HMB45 20X 


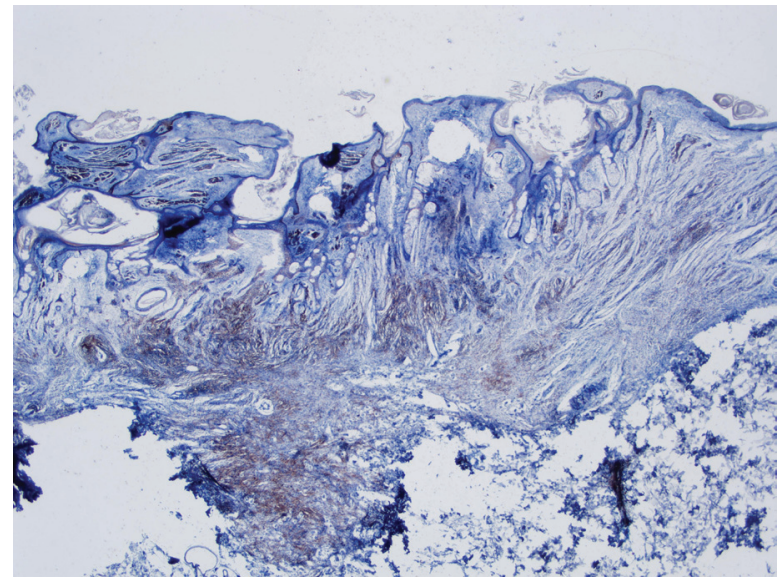

Figure 2b: IHC showing negative stain for MelanA 20X

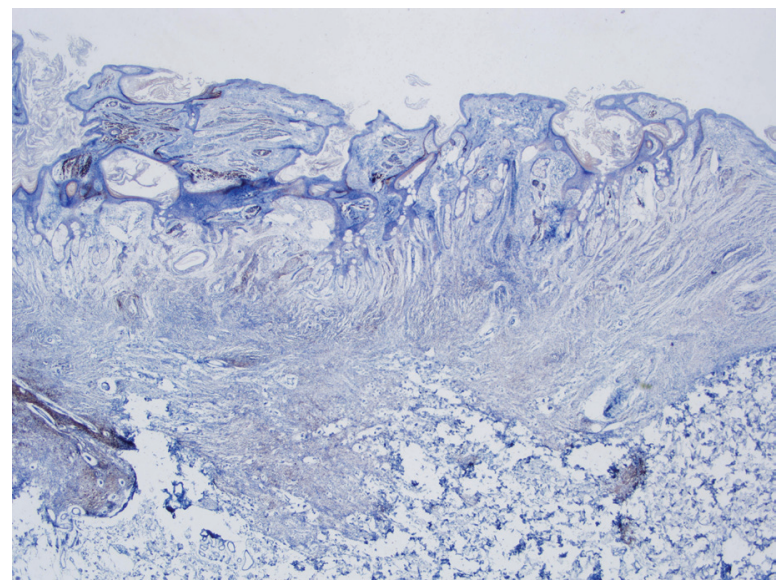

Figure 2d: IHC showing negative stain for MITF 20X

looked nevus. But, after histopathologic examination, it came out to be a puzzling case of two histologically distinct tumors coinciding at the same location. The present case is the second case of FEP and CMN coinciding at the same location, which makes it a rare case. Both FEP and CMN further needs detail laboratory

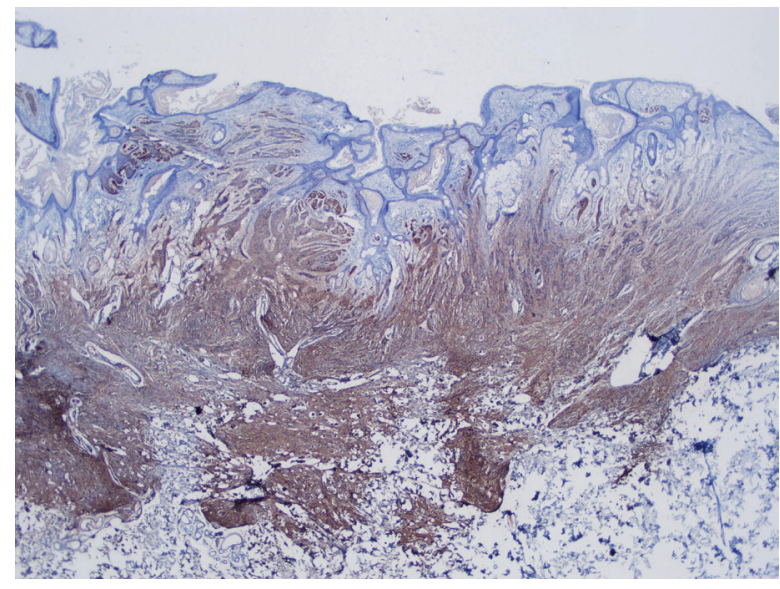

Figure 2c: IHC showing negative stain for S100 20X

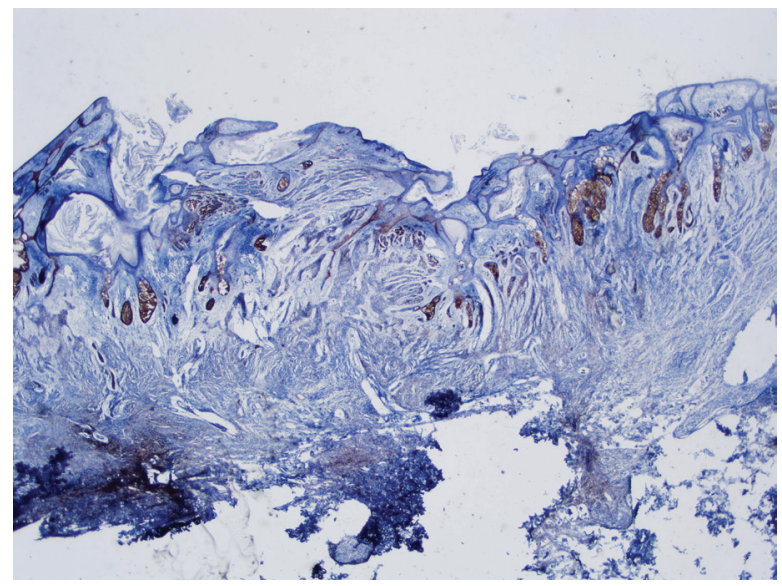

Figure 2e: IHC showing negative stain for EMA 20X

and radiological work up to rule out malignancy. Hence, this case emphasizes the importance of detailed histopathologic examination in order to make correct diagnosis and detect malignancy, if present, at earlier stage.

\section{References}

1. Sunassee A, Kerkvliet AM, Jassim AD. Combined Melanocytic Nevus, superficial congenital and deep penetrating Types with Fibroepithelioma of Pinkus, Collision Tumor A Case Report. S D Med. 2017; 70: 363-365.

2. Warner TF, Burgess $\mathrm{H}$, Mohs FE. Extramammary Paget's disease in fibroepithelioma of Pinkus. J Cutan Pathol. 1982; 9: 340-344. https://doi. org/10.1111/j.1600-0560.1982.tb01070.x

3. Bryant J. Fibroepithelioma of Pinkus overlying breast cancer. Arch Dermatol. 1985; 121: 310. https://doi.org/10.1001/archderm.121.3.310

4. Sandbank M, Baschan D: Multiple trichoepitelioma and breast carcinoma. Simultaneous appearance of epithelioma adenoides cysticum and infiltrating lobular carcinoma of the breast. Arch Dermatol 1978; 114: 1230. https://doi.org/10.1001/ archderm.114.8.1230

5. Longo C, Pellacani G, Tomasi A, Mandel VD, Ponti G. Fibroepithelioma of Pinkus: Solitary tumor or sign of a complex gastrointestinal syndrome. Mol Clin Oncol. 2016; 4: 797-800. https://doi. org/10.3892/mco.2016.794

6. Gorlin RJ and Goltz RW: Multiple nevoid basal-cell epithelioma, jaw cysts and bifid rib. A syndrome. N Engl J Med. 1960; 262: 908-912. https://doi. org/10.1056/NEJM196005052621803

7. Haddock ES, Cohen PR. Fibroepithelioma of Pinkus Revisited. Dermatol Ther. 2016; 6:347-62. https://doi.org/10.1007/ s13555-016-0123-8 